\title{
Antigen-based Rapid Diagnostic Testing or Alternatives for Diagnosis of Symptomatic COVID-19
}

\author{
A Simulation-based Net Benefit Analysis
}

\author{
Emily A. Kendall, ${ }^{a}$ Nimalan Arinaminpathy, ${ }^{\mathrm{b}}$ Jilian A. Sacks, ${ }^{\mathrm{c}}$ Yukari C. Manabe, ${ }^{\mathrm{a}}$ Sabine Dittrich, ${ }^{\mathrm{c}}$ \\ Samuel G. Schumacher, ${ }^{\mathrm{c}}$ and David W. Dowdy ${ }^{\mathrm{d}}$
}

\begin{abstract}
Background: SARS-CoV-2 antigen-detection rapid diagnostic tests can diagnose COVID-19 rapidly and at low cost, but lower sensitivity compared with reverse-transcriptase polymerase chain reaction (PCR) has limited clinical adoption.

Methods: We compared antigen testing, PCR testing, and clinical judgment alone for diagnosing symptomatic COVID-19 in an outpatient setting (10\% COVID-19 prevalence among the patients tested, 3-day PCR turnaround) and a hospital setting (40\% prevalence, 24-hour PCR turnaround). We simulated transmission from cases and contacts, and relationships between time, viral burden, transmission, and case detection. We compared diagnostic approaches using a measure of net benefit that incorporated both clinical and public health benefits and harms of the intervention.
\end{abstract}

Results: In the outpatient setting, we estimated that using antigen testing instead of PCR to test 200 individuals could be equivalent to preventing all symptomatic transmission from one person with COVID-19 (one "transmission-equivalent"). In a hospital, net benefit analysis favored PCR and testing 25 patients with PCR instead of antigen testing achieved one transmission-equivalent of benefit. In both settings, antigen testing was preferable to PCR if PCR turnaround time exceeded 2 days. Both tests provided greater net benefit

Submitted January 29, 2021; accepted July 2, 2021

From the aDivision of Infectious Diseases, Johns Hopkins University School of Medicine, Baltimore, MD; 'bepartment of Epidemiology, Johns Hopkins Bloomberg School of Public Health, Baltimore, MD; ${ }^{\mathrm{M}} \mathrm{MRC}$ Centre for Global Infectious Disease Analysis, Imperial College, London, United Kingdom; and ${ }^{\mathrm{d} F o u n d a t i o n}$ for Innovative New Diagnostics, Geneva, Switzerland.

S.G.S. and D.W.D. have contributed equally as co-senior authors.

The results reported herein correspond to specific aims of award CV20-0022 from Foundation for Innovative New Diagnostics (FIND), through funding from the World Health Organization. This work was also supported by grants U54EB007958-12, U5411090366, and 3U54HL143541-02S2 from the National Institutes of Health.

Model code is available at https://github.com/eakendall/DCA. Data are from published sources as cited

J.A.S., S.D., and S.G.S. are employed by the Foundation for Innovative New Diagnostics (FIND). The other authors have no conflicts to report.

SDC Supplemental digital content is available through direct URL citations SDC in the HTML and PDF versions of this article (www.epidem.com).

Correspondence: Emily A. Kendall, Division of Infectious Diseases, Johns Hopkins University School of Medicine, 1550 Orleans St, Rm 106, Baltimore, MD 21287. E-mail: ekendall@jhmi.edu.

Copyright $@ 2021$ Wolters Kluwer Health, Inc. All rights reserved. ISSN: $1044-3983 / 21 / 326-811$

DOI: $10.1097 / E D E .0000000000001400$ than management based on clinical judgment alone unless intervention carried minimal harm and was provided equally regardless of diagnostic approach.

Conclusions: For diagnosis of symptomatic COVID-19, we estimated that the speed of diagnosis with antigen testing is likely to outweigh its lower accuracy compared with PCR, wherever PCR turnaround time is 2 days or longer. This advantage may be even greater if antigen tests are also less expensive.

Keywords: SARS-CoV-2; Diagnostic tests; Symptomatic outpatients; Hospitals; Rapid antigen tests; Simulation; Decision-curve analysis

(Epidemiology 2021;32: 811-819)

A ccurate diagnosis of COVID-19 can guide clinical management, reduce transmission, and inform appropriate allocation of resources for isolation, contact tracing, and treatment. In many settings - including low- and middle-income countries (LMICs) as well as high-income countries with large outbreaks - efforts to diagnose COVID-19 using reverse-transcriptase polymerase chain reaction (PCR) and other nucleic acid amplification tests frequently exceed capacity. ${ }^{1-3}$

Antigen-detection rapid diagnostic tests ("antigen tests") are less expensive than PCR and can be performed in minutes without centralized laboratory infrastructure. Thus, they could facilitate higher volumes of testing and provide rapid results, while also relieving strains on laboratory capacity. Antigen tests are, however, less sensitive than PCR. Some experts have argued that all SARS-CoV-2 testing must be highly sensitive, ${ }^{4,5}$ while others advocate less sensitive testing primarily for community-level surveillance. ${ }^{6}$ Recognizing the limited capacity for PCR in many settings, WHO and national public health agencies have issued target product profiles for antigen tests ${ }^{7}$ and interim guidance for their use in select circumstances. ${ }^{8-10}$ Meanwhile, a global partnership has begun to manufacture and distribute 120 million antigen tests in LMICs. ${ }^{11}$

As antigen tests become more widely available, it is important to understand the conditions under which their implementation would be preferable to relying on PCR or clinician judgment alone. This understanding must balance 
accuracy (i.e., sensitivity and specificity) with other considerations such as the turnaround time for results and the likely actions in response to a positive (or negative) diagnostic result. We sought to aid decision-making by quantifying these tradeoffs into a single unified measure of net benefit when used for patients presenting with symptomatic COVID-19 in the inpatient and outpatient settings.

\section{METHODS}

\section{Overview}

We developed a simulation model of COVID-19 diagnostic evaluation, in which we compared the use of point-ofcare antigen testing, centralized (e.g., hospital-based) PCR testing, and clinical judgment (i.e., without virologic testing) among individuals presenting with symptoms suggestive of COVID-19. We parameterized the model using published data (and assumptions, where published data did not exist) regarding SARS-CoV-2 viral dynamics, clinical features of COVID-19, and diagnostic assay performance. The model accounts for the following aspects of disease dynamics and diagnostic testing: (1) time-dependent transmission from index cases and their contacts (Figure 1A); (2) variable timing of clinical presentation; (3) nonuniform distribution of peak viral burden across the population (Figure 1B); (4) correlation of viral burden with both assay sensitivity (Figure 1B, dotted horizontal lines) and infectivity (eFigure 1; http://links.lww.com/EDE/B834); and (5) assay-dependent decline in sensitivity with time since symptom onset (Figure 1C). After simulating these dynamics and their effects on transmission-related and clinical outcomes, we used an adaptation of net benefit analysis ${ }^{12-14}$ to combine the transmission-related benefits, the clinical benefits, and the harms of intervention after diagnosis on a single net benefit scale with units of "transmission-equivalents," where $1.0=$ equivalent benefit to averting all symptomatic transmission from an average person with symptomatic COVID-19. We then used this scale to characterize the incremental net benefit of antigen testing relative to either PCR or clinical judgment alone, under a variety of assumptions. While costs can also be included in the calculation of net benefit, this requires monetization of clinical and transmission benefits; thus, we elected to exclude costs from this analysis.

\section{Simulated Patient Populations and Testing Strategies}

We separately simulated two symptomatic patient populations being evaluated for COVID-19: patients with moderate-to-severe illness in a hospital setting (of whom $40 \%$ had COVID-19) and mildly symptomatic people in an outpatient setting (with more nonspecific symptoms, of which $10 \%$ represented true COVID-19 ${ }^{15}$ ). As described in the eAppendix; http:// links.lww.com/EDE/B834, other differences between the two settings included a longer turnaround time for PCR in the outpatient setting ( 1 day in the hospital, 3 days for outpatients ${ }^{16,17}$ ), a greater amount of presumptive isolation while awaiting diagnostic results in the hospital setting, a longer mean duration of symptoms before presentation in the hospital setting, and a greater value placed on clinical improvement in the hospital setting versus reduction of transmission in the outpatient setting. These features were all evaluated in sensitivity analyses.

Antigen tests were assumed to have lower sensitivity at symptom onset (85\% relative to PCR, eTable 1; http:/links. lww.com/EDE/B834), with the highest probability of detecting those with the highest viral burdens.

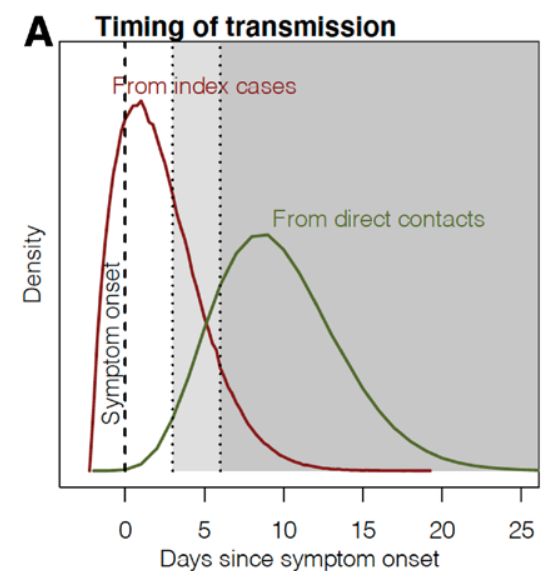

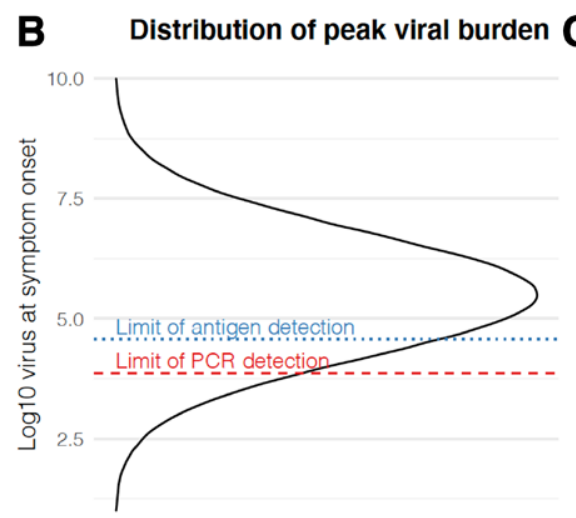

Proportion of patient population

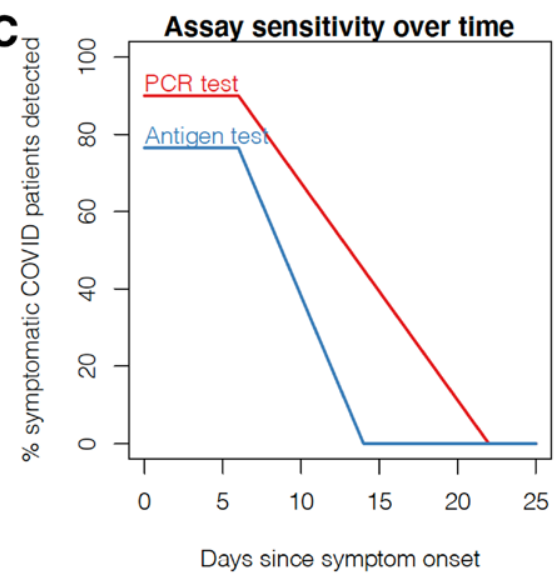

FIGURE 1. Key assumptions in simulation of net benefit. (A) The timing of transmission events both from cases and from their direct contacts, in absence of intervention, follows a nonuniform distribution relative to the time of symptom onset in the index case. Shading illustrates that different amounts of transmission are preventable after earlier (light shading) versus later (dark shading) diagnosis. (B) The simulated viral burden in upper respiratory diagnostic specimens at symptom onset is log-normally distributed across a simulated population. This may be conceptualized in terms of genome copies on a quantitative reverse-transcriptase PCR (units shown on y axis) or antigen copies (assumed to vary in proportion to genome copies during acute illness). (C) After an initial period of maximal detection, the simulated sensitivity of antigen testing declines on a similar timeline as infectivity, and sensitivity of PCR declines more slowly. 
In addition to comparing antigen and PCR testing, we compared both virologic assays to a clinical (i.e., nonvirologic) diagnostic approach. Clinician judgment was estimated to have $80 \%$ sensitivity (comparable, at the time of clinical presentation, to the proportion of patients detectable by PCR), with corresponding $50 \%$ specificity ${ }^{18}$ (compared with $99.5 \%$ and $99 \%$, respectively, for PCR and antigen testing, eTable 1; http://links.lww.com/EDE/B834). We assumed that clinical diagnoses, being less certain, may result in less intense intervention - for example, less stringent isolation, less case reporting or contact notification, or more circumspect clinical management-and therefore reduced both the benefit and harm of intervention (by a factor of 0.75 in the primary analysis), compared with virological diagnoses made using antigen or PCR testing. For directness of comparison, we assumed that only a single diagnostic approach would be used in a given setting and patient population, with no further testing or clinical risk stratification after the initial evaluation.

\section{Simulated SARS-CoV-2 Transmission Dynamics and Diagnosis}

Our model stochastically simulated the timing of transmission events from index cases and their contacts relative to index cases' symptom onset (Figure 1A; details in eAppendix; http://links.lww.com/EDE/B834). The model accounted for presymptomatic transmission, ${ }^{19-21}$ delays between symptom onset and clinical presentation, ${ }^{22-24}$ partial isolation during the wait for awaiting test results, and more stringent (but still imperfect) isolation and contact tracing/quarantine once a COVID-19 diagnosis was made. ${ }^{25,26}$

We assumed that viral burden coincided with the onset of symptoms and was log-normally distributed in the population (Figure 1B), ${ }^{27,28}$ and that both diagnostic sensitivity and infectivity were functions of this viral burden. Specifically, based on observed relationships between PCR cycle threshold, viral culture, and infectivity, ${ }^{22,29-31}$ and similar to other models, ${ }^{6}$ we assumed a log-linear relationship between peak viral burden and peak infectivity, above a minimum threshold of $10^{3}$ viral genome copies. We also assumed that for both antigen and PCR testing, infected patients who tested positive had higher viral burdens than those who tested negative (Figure 1B). Thus, infectivity was greater for PCR-positive/ antigen-positive patients than for PCR-positive/antigennegatives (eFigure 1; http://links.lww.com/EDE/B834). We also assumed that assay sensitivity declined over time after an initial 6-day period of maximal sensitivity; antigen test sensitivity was assumed to decline on a similar timeline as infectivity, while PCR sensitivity declined more slowly and thus detected some patients who were no longer infectious (Figure 1C; details in eAppendix; http://links.lww.com/EDE/ B834). ${ }^{22,32-34}$

Using these assumptions, we simulated a population of index patients with COVID-19, each with a specified peak viral burden and time to clinical presentation. We chose to simulate a large population of 1,000,000 index patients to reduce stochastic variability and provide more reliable estimates of mean behavior (eTable 4; http://links.lww.com/EDE/ B834). (Additional patients without COVID-19 enter our analysis at a later step when we estimate the harms of falsepositive results.) We simulated the timing of first-generation transmission events from index patients to their direct contacts (according to index patients' relative infectivity) and of second-generation transmission events from index patients' contacts to contacts of their own, in absence of intervention. We then simulated the result of each diagnostic test, based on each patient's peak viral burden, the time from symptom onset to testing, and assay sensitivity at that time (Figure 1C). Finally, we simulated the effects of case isolation and contact quarantine as probabilities of averting first- and second-generation transmission events, respectively (eTable 2; http://links. lww.com/EDE/B834).

We also modeled the clinical impact of COVID-19specific medical treatments on morbidity and mortality as time-sensitive, declining exponentially with time since symptom onset. We assumed that the average impact of treatment was much greater in the hospital than the outpatient setting (eAppendix section 2.1; http://links.lww.com/ $\mathrm{EDE} / \mathrm{B} 834)$.

\section{Estimating Benefit of Diagnostic Approaches}

We created a unified measure of the combined value ("net benefit") of each testing strategy, equal to (a) the public health benefit of preventing transmission through case isolation and contact quarantine, plus (b) the clinical benefit of preventing morbidity and mortality through COVID-19-specific treatment, minus (c) the harms of these interventions. To combine these quantities on a common scale, we placed these benefits and harms on a scale of "transmission-equivalents," where a value of 1.0 equals the value of preventing all symptomatic transmission from one patient of average infectiousness who develops symptoms. Our novel approach builds on decisioncurve analysis, in which benefits and harms of intervention are estimated on a single scale using a weighting factor. ${ }^{14,34,35}$ Two novel ways in which our approach builds on this framework are (i) separately estimating clinical and public health benefits in a mechanistic way, and (ii) capturing how benefit may vary depending on the patient or the timing of diagnosis. In doing so, our analysis captures key tradeoffs between the accuracy and timeliness of diagnosis, tradeoffs that are inherent in comparing antigen testing with PCR. Our analysis also differs from the conventional approach in that it incorporates potential harms of intervention (e.g., inconvenience of isolation, side effects of treatment) for both true-positive and falsepositive diagnoses.

We estimated transmission-related benefits as a weighted average of the proportion of transmission that was prevented from index cases (through isolation) and from 
contacts (either by preventing infection or through contact quarantine) (eAppendix Section 1.6; http://links.lww. $\mathrm{com} / \mathrm{EDE} / \mathrm{B} 834)$. This quantity was estimated relative to the total amount of transmission that was modeled to occur after symptom onset, to estimate benefit in units that we call transmission-equivalents.

We estimated clinical benefits by first estimating the effectiveness of a given diagnostic approach at averting avertible mortality, accounting for delays in diagnosis and treatment and for imperfect test sensitivity (eAppendix Section 2.1; http://links.lww.com/EDE/B834). This benefit was converted to transmission-equivalent units by estimating the number of cases from whom all symptomatic transmission (to average-risk individuals) would need to be prevented, to achieve the same expected mortality reduction as would be achieved through prompt treatment of one symptomatic case in the setting under consideration (eAppendix Section 2.1; http://links.lww.com/EDE/B834).

We multiplied the estimated per-patient transmissionrelated and clinical benefits by the prevalence of COVID in the population (i.e., assuming no benefit to patients who had symptoms but no underlying COVID-19), to determine the benefits of testing per patient tested. These benefits were then reduced by an estimate of the per-patient harms of testing and resulting intervention (e.g., inconvenience of isolation or side effects of treatment). The harms were assumed to apply to every patient with a positive diagnostic result regardless of whether that patient had underlying COVID-19. To place harms on the same transmission-equivalent scale as benefits, we used an approach from decision-curve analysis: ${ }^{35}$ we defined a threshold probability $q$ of a patient having a condition (in this case, COVID-19), above which one would isolate a newly symptomatic patient and expect the benefits to outweigh harms. In our formulation, this corresponds to an assumption that the benefit of intervening promptly on a patient with COVID-19 is $1 / q$ times as large as the harm of intervening on any patient (irrespective of COVID-19 status); for purposes of our analysis, therefore, we refer to this quantity $q$ as the harm-benefit ratio. We estimated $q$ at 0.1 in the reference scenario based on the observed willingness to isolate or quarantine individuals in current practice (eAppendix Section 2.2; http://links.lww.com/EDE/B834), but as is standard in decision-curve analysis, we also considered results across a range of harm-benefit ratios. The resulting net benefit equation is presented and explained in eAppendix Section 2.3; http://links.lww.com/EDE/B834.

In these analyses, we estimated the net benefit of each diagnostic approach and the incremental net benefit of antigen testing relative to PCR. We also present decision curves that show the net benefit of each strategy according to the harmbenefit ratio $q$.

This research did not involve human subjects and did not require ethical review. We performed all analyses using $\mathrm{R}$ version 4.0.2.

\section{RESULTS}

\section{Transmission-related Benefits of Diagnosis}

The simulated number and timing of transmission events, under each diagnostic scenario, are shown in Figure 2. In the outpatient setting, an estimated $63 \%$ of COVID-19 cases were detected under the antigen-testing scenario, compared with $80 \%$ for both PCR testing and clinical judgment (Figure 2A); in the hospital setting, later presentation slightly reduced these percentages, to $57 \%$ and $75 \%$, respectively (Figure 2B). However, antigen testing detected cases more rapidly than PCR (Figure 2A,B), and it also detected the cases who were most infectious (for example, the $63 \%$ of patients who were antigen-detectable accounted for $77 \%$ of all subsequent transmission events). These effects led to prevention of similar amounts of transmission by antigen testing (blue line, Figure 2C,D), PCR (red line, Figure 2C,D), and clinical judgment alone (with less intensive intervention; green line, Figure 2C,D). In the hospital setting, PCR remained the most effective approach for averting transmission (Figure 2D), but in the outpatient setting (Figure 2C), we estimated that antigen testing could avert $32 \%$ of all transmission, compared with 29\% for PCR and 31\% for clinical judgment alone.

\section{Net Benefit, Incorporating Harms, and Clinical Benefits of Diagnosis}

Figure 3 shows estimates of net benefit for antigen testing relative to PCR, under different scenarios of PCR turnaround time and antigen test sensitivity (relative to PCR). In the outpatient setting, we estimated the net benefit provided by either PCR or antigen testing, relative to no testing, to be between 0.02 and 0.04 transmission-equivalents (Figure 3A,B, lower dotted lines). Thus, 25 to 50 individuals would need to be tested to achieve a net benefit equivalent to preventing all symptomatic transmission from one average case with symptoms. In the hospital setting, where the prevalence of COVID-19 was higher and the potential benefits of prompt treatment much greater, the net benefit of testing (relative to no testing) ranged from 0.3 to 0.6 transmission-equivalents (figure $3 \mathrm{~A}, \mathrm{~B}$, upper solid lines). Thus, between two and three tests in the hospital were needed to achieve the same net benefit as 25 to 50 tests in the outpatient setting.

In both settings, the points at which antigen testing provided incremental net benefit relative to PCR (i.e., points of intersection in Figure 3A, "0" contour lines in Figure 3C,D) were sensitive to the turnaround time for PCR results (Figure 3A) and, to a lesser degree, the sensitivity of antigen tests (Figure 3B). At our primary estimates of antigen test sensitivity ( $85 \%$, defined acutely relative to PCR), antigen testing provided an incremental net benefit of 0.005 transmissionequivalents relative to $\mathrm{PCR}$ in the simulated outpatient setting with an assumed 3-day PCR turnaround time (Figure 3C, asterisk), whereas PCR was preferred and provided an incremental net benefit of 0.04 transmission-equivalents relative to antigen testing in the simulated hospital with an assumed 1-day PCR 
A

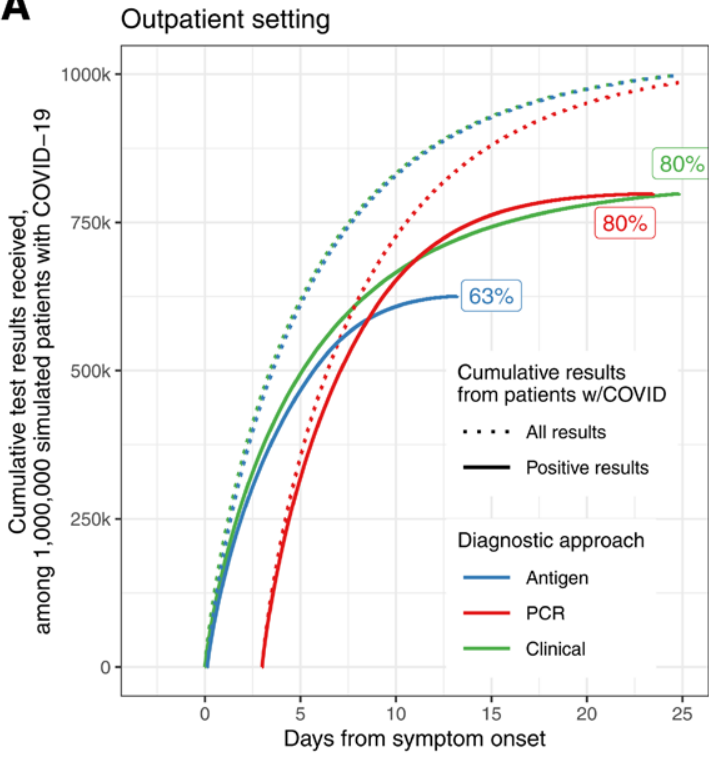

C

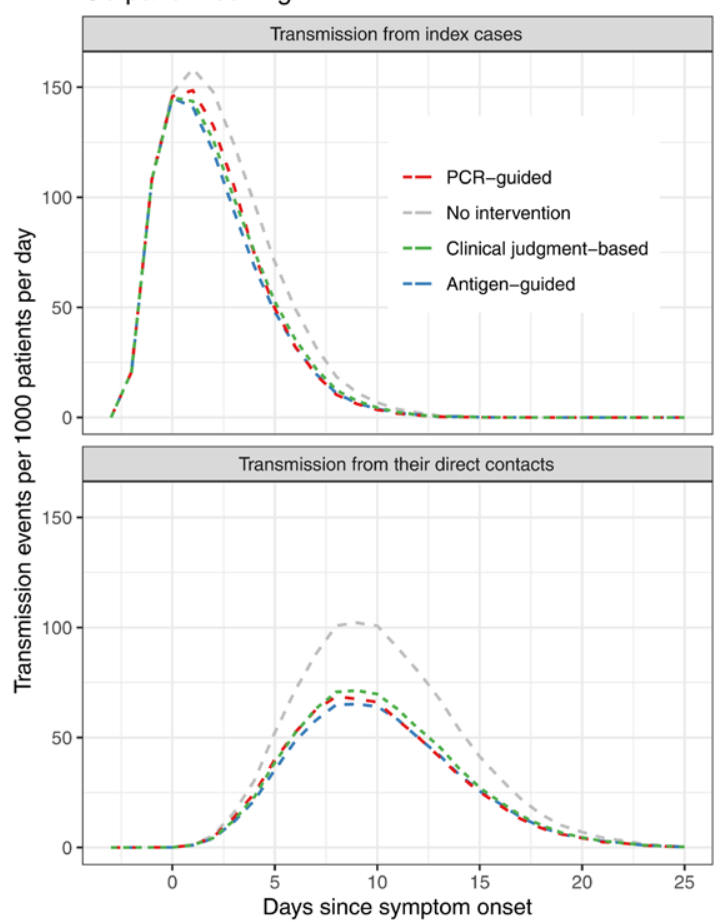

B

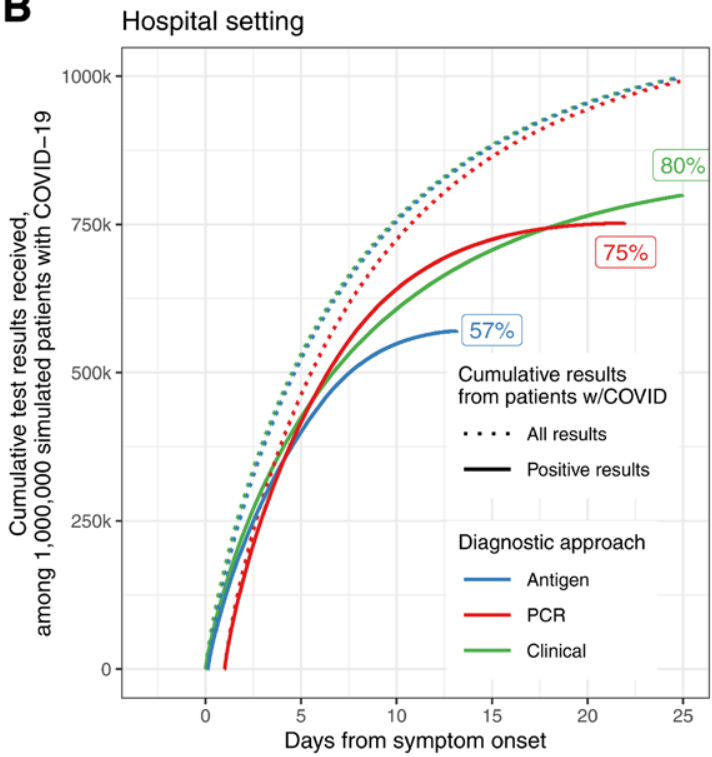

D Hospital setting

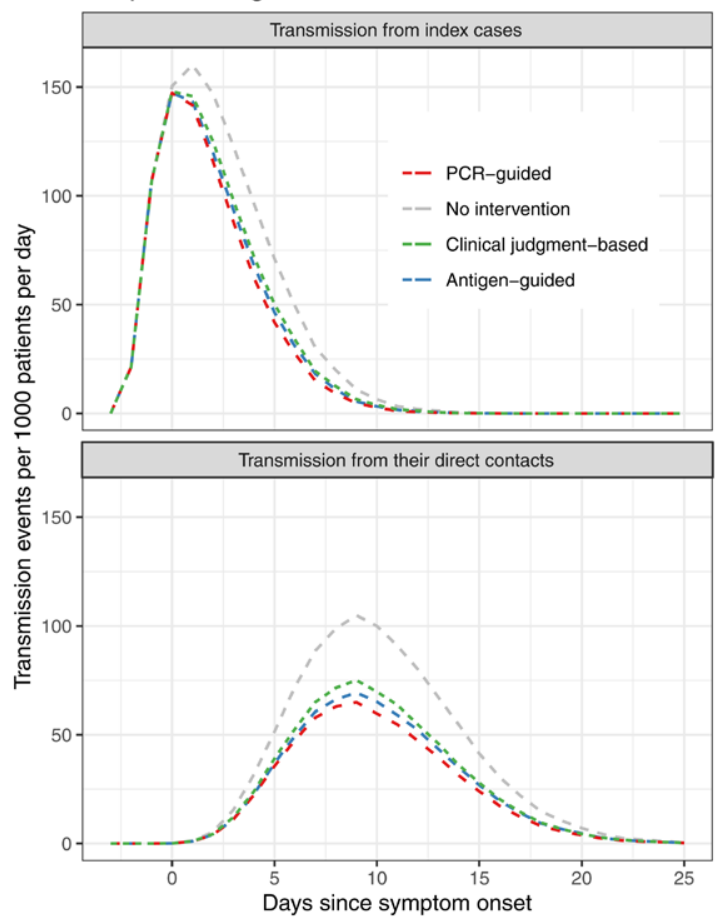

FIGURE 2. Timing of testing and SARS-CoV-2 transmission events under different diagnostic algorithms in the outpatient setting. (A, B) Cumulative test results over time among 1,000,000 patients with COVID-19 in the (A) outpatient and (B) hospital settings. Time of symptom onset and clinical presentation ( 3 days after symptom onset) is aligned for all patients; thus, PCR turnaround time is depicted as the space on the $x$-axis before the start of the red curves. Solid lines indicate all results received (including falsenegative results) from patients with COVID, and dashed lines indicate only the positive results. Antigen tests and clinical judgment provide same-day results (and thus have curves shifted to the left) relative to PCR but PCR ultimately detects more true-positive COVID-19 cases than antigen testing (80\% versus 63\%). Clinical judgment may diagnose as many true COVID-19 cases as PCR but with lower specificity and no preference for the most infectious cases (not shown). (C, D) Quantity and timing of transmission with and without intervention in the (C) outpatient and (D) hospital settings. Delayed clinical presentation, incomplete isolation, and incomplete contact notification/quarantine prevent any testing strategy from preventing the majority of transmission (difference between gray lines and colored lines). Antigen testing (blue lines) achieves slightly greater reductions in transmission in the outpatient setting where PCR delays are long, whereas PCR (red lines) achieves slightly greater reduction in the hospital, where the benefit of greater sensitivity outweighs the negative effect of a 1-day delay in results. 
A

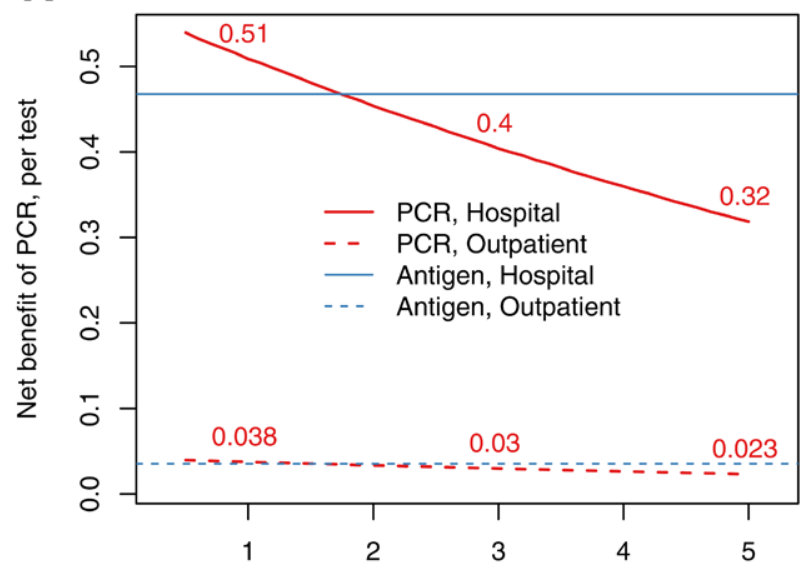

PCR turnaround time (days)

\section{C}

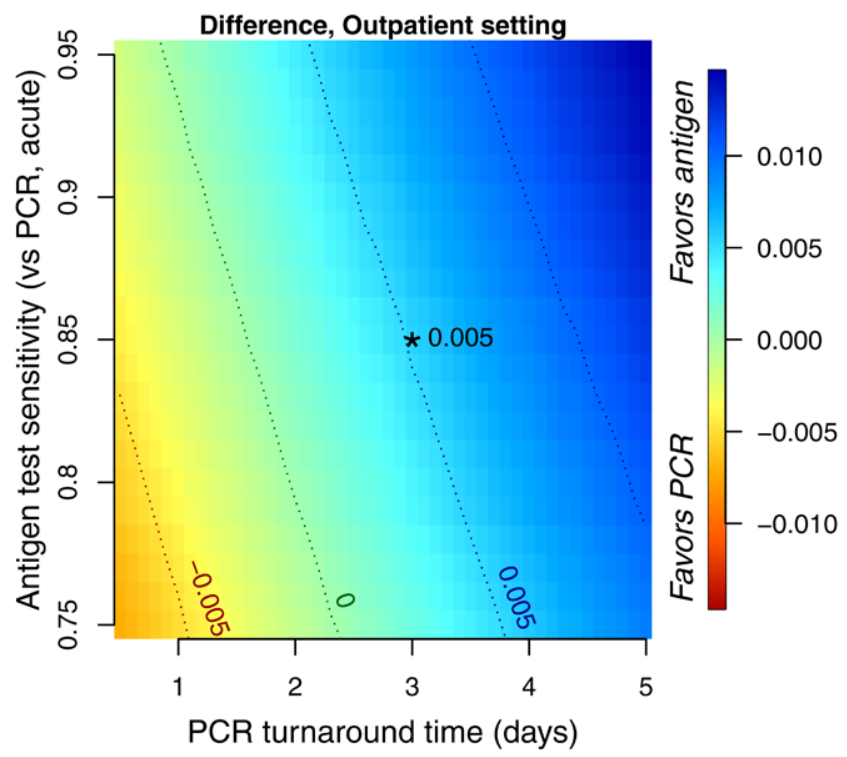

B
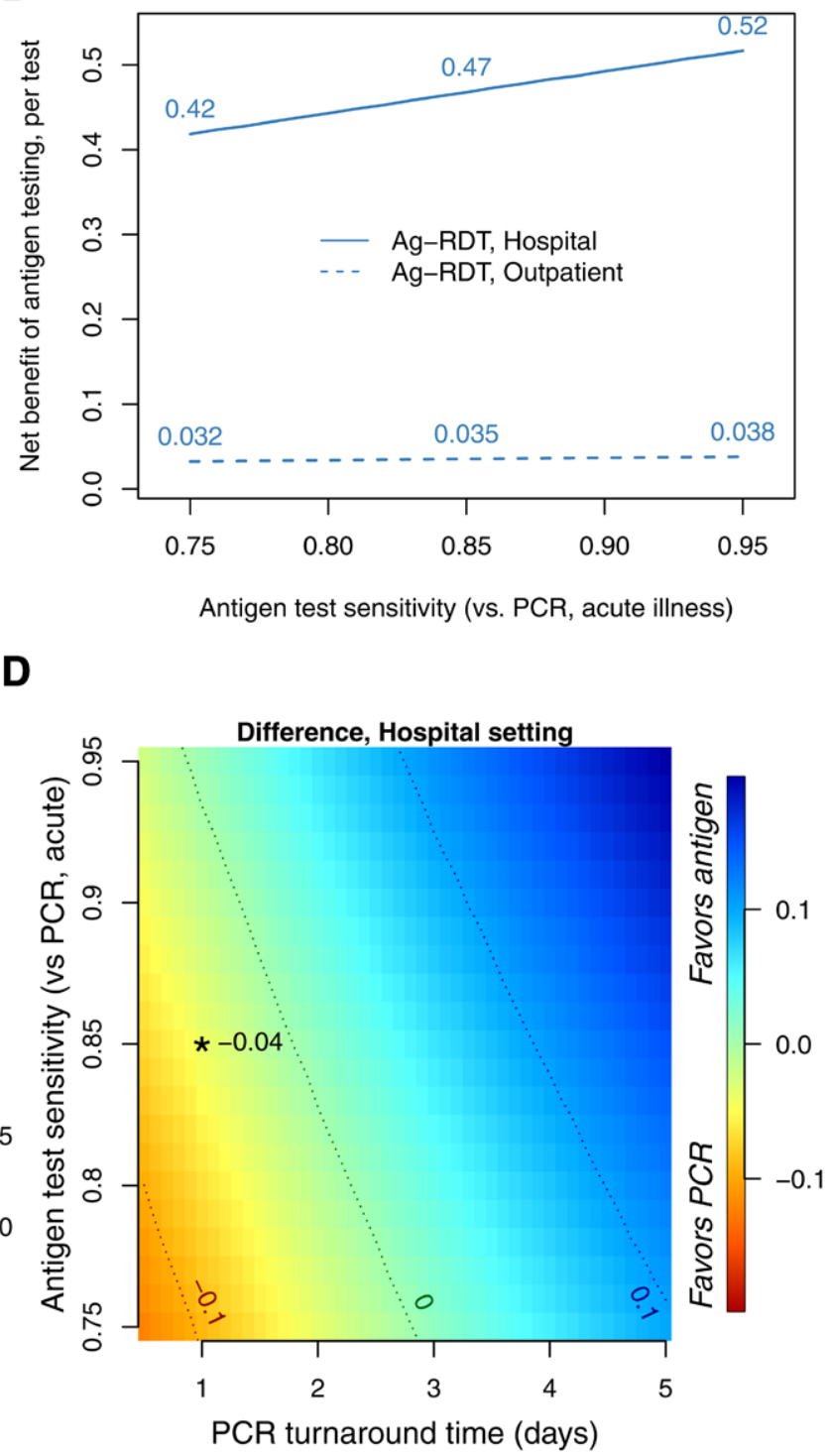

FIGURE 3. Net benefit of COVID-19 testing strategies. (A, B) show the net benefit of testing each patient population with PCR $(A$, red) rapid antigen tests $(B$, blue) over a range of values for PCR turnaround time (A) or assay sensitivity (B). Net benefit is expressed in "transmission-equivalents" per patient tested, with 1 unit equivalent to the value of preventing all symptomatic transmission from one average case. Points of intersection in (A) indicate equivalent net benefit for the two testing strategies, holding all other parameters at their reference values. (C, D) show the difference in net benefit between the two assays (antigen minus PCR) in the outpatient setting (C) and hospital setting (D). The colors scales differ by setting, due to the larger magnitude of net benefit per patient tested, for either test, in the hospital setting. Asterisks ${ }^{*}$ ) indicate the net benefit associated with our primary estimates of PCR turnaround time and antigen test sensitivity for each setting, and contour lines indicate parameter value combinations that produce the same specified incremental net benefit.

turnaround time (Figure 3D, asterisk). In both settings, with an assumed antigen test sensitivity of $85 \%$ relative to PCR, antigen testing was the preferred strategy (i.e., positive incremental net benefit) when PCR turnaround times were $\geq 2$ days. This threshold for equivalency dropped to 1 day if antigen testing was assumed to have $95 \%$ sensitivity, and increased to 3 days if antigen testing was assumed to have $75 \%$ sensitivity, relative to PCR (Figure 3C,D, “0” contour lines).
Our finding that antigen testing offered positive incremental net benefit relative to PCR in the outpatient setting was robust to variation in most other parameter values (eFigure 4A; http://links.lww.com/EDE/B834). One exception was isolation practices while awaiting test results: if strict isolation (i.e., sufficient to reduce transmission by $70 \%$ ) were maintained during the wait for PCR results, without any additional harms, then PCR would be preferred to antigen testing 
in this setting. In the hospital setting, antigen testing could offer greater net benefit than PCR if clinical interventions were highly time-sensitive (such that the morbidity and mortality that remained avertable by treatment decreased by $30 \%$ per day). Contributions of prevented transmission, prevented mortality, and false-positive diagnoses to the net benefit are explored in eTable 3; http://links.lww.com/EDE/B834.

As a final consideration, we estimated the change in net benefit of each assay as we varied the harm of intervening on a positive result relative to the benefit of prompt intervention in a patient with COVID-19 (i.e., varied the threshold probability for treatment; eFigure 5; http://links.lww.com/EDE/ B834). At high-relative harms of intervention, antigen testing is increasingly preferred over PCR, because the harms of intervening on PCR-positive/antigen-negative individuals (who are less infectious than average or late in their disease course) become large relative to the benefits. By contrast, at very small estimates of relative harm, clinical judgment - with high sensitivity but low specificity and thus high probability of false-positive diagnosis - becomes increasingly favored. However, the net benefit of clinical judgment was projected to exceed that of PCR or antigen testing only if clinically diagnosed patients could receive interventions of equal intensity as patients with a positive virological test - and even then, only if the harm of such intensive intervention was deemed equivalent to $10 \%$ or less of the benefit of intervening on one person with true COVID-19.

\section{DISCUSSION}

As antigen-detection SARS-CoV-2 rapid diagnostic tests become more widely available, it is important to identify the settings under which they offer incremental benefit over PCR testing or clinical judgment alone. We developed a novel adaptation of net benefit analysis that accounts for both the clinical and transmission-related benefits of diagnosis in clinical settings, as well as the harms of intervening (on both false-positives and true-positives). Using this framework, we demonstrate that antigen testing is likely to yield greater health benefit than PCR under typical outpatient conditions that include turnaround times of $\geq 2$ days for PCR results, even if antigen tests have considerably lower sensitivity. We demonstrate that antigen testing would be similarly favored over PCR in a hospital setting with PCR turnaround times of 2 days or more-although faster turnaround times are likely to be available in many hospitals. Furthermore, an antigen-based assay that achieved sensitivity $>95 \%$ relative to PCR during acute illness could provide greater benefit than PCR even at PCR turnaround times of 1 day. Finally, we also demonstrate that both PCR and antigen testing are preferable over clinical judgment alone, under reasonable assumptions about the harms of false-positive diagnoses and the likely intensity of response to virologically unconfirmed diagnoses.

Compared with other estimates of the transmission burden that might be prevented through frequent asymptomatic screening, ${ }^{37-39}$ our results suggest that symptom-driven testing may have limited potential to reduce transmission because of late clinical presentation relative to the onset of infectiousness (one exception, not modeled here, might be settings with extensive backward contact tracing after a symptom-based diagnosis ${ }^{40,41}$ ). Our results also show that testing alone will avert only a small proportion of poor clinical outcomes due to the limited efficacy of COVID-specific treatments and the frequent empiric provision of nonspecific treatments. Nevertheless, our comparisons of PCR and antigen testing in clinical settings align with models comparing the same tests for asymptomatic screening. ${ }^{6,38}$ Specifically, our results indicate that rapid results can overcome the disadvantages of suboptimal sensitivity and allow the net benefit of antigen testing to match or (when PCR turnaround times are long) exceed that of slower but more sensitive PCR testing.

Our novel application of net benefit and decisioncurve analysis to COVID-19 demonstrates the importance of accounting for factors beyond sensitivity and specificityincluding the ability to diagnose the most epidemiologically important and clinically treatable cases-when evaluating infectious disease diagnostics. Incorporating all of these considerations into a single metric of net benefit, our analysis suggests that antigen testing is likely to produce better outcomes than PCR with $\geq 2$ day PCR turnaround time, as is common in outpatient settings, whereas PCR is likely to be preferred in facilities with PCR capacity that can achieve same-day or next-day turnaround. Conventional decision-curve analysisbased only on sensitivity, specificity, prevalence, and threshold probability - would incorrectly favor the more accurate test (i.e., PCR) in all settings, even when the timing of diagnosis is critical.

Although any model-based findings are subject to bias, our estimates are likely to be conservative regarding the benefit of adopting antigen testing in clinical settings. First, our analysis does not incorporate the lower economic cost of antigen tests relative to PCR - although these costs may be partially offset by additional follow-up tests for SARS-CoV-2 or alternative diagnoses that may be required after false-negative antigen results. Second, our primary estimates of antigen test sensitivity in the acute phase (i.e., $85 \%$ relative to PCR) may be conservative. ${ }^{22}$ Third, our model does not account for the potential that patients who test falsely negative by antigen tests may still receive some degree of isolation or clinical intervention on the basis of high-clinical suspicion.

Our model is limited by underlying data availability about SARS-CoV-2 dynamics. In particular, data on the relationship between viral burden and infectivity remain sparse. Our ability to draw conclusions that apply across settings and assays is also limited by varying clinical and public health practice - for instance, the extent to which symptomatic people self-isolate or contact tracing is performed are likely to vary widely across settings. Estimates of threshold probability are also context specific, depending, for example, on the 
individual and societal economic costs of interventions such as contact investigation and quarantine in each particular setting. Third, certain parameter estimates - such as the importance of preventing downstream transmission relative to preventing poor clinical outcomes in people currently infected - depend on prevailing epidemic trends more broadly. Preventing transmission may be more important in settings with emerging or widespread transmission and less important in settings with resolving epidemics or imminent widespread vaccination. Finally, our analysis focuses on diagnosis of symptomatic individuals and quarantine of their direct contacts and does not consider the potential role of antigen testing in preventing presymptomatic or asymptomatic transmission.

In conclusion, this novel application of net benefit analysis demonstrates that, for individuals with symptoms suggestive of COVID-19, rapid SARS-CoV-2 testing using an antigen test with $85 \%$ sensitivity (measured relative to PCR and during acute symptoms) could offer greater net benefit than either PCR or clinician-driven diagnosis. We identified a threshold near 2 days for the turnaround time of PCR results, above which antigen testing is expected to offer greater net benefit than PCR. Use of antigen testing may be particularly beneficial in outpatient settings - where testing volumes are often high, PCR turnaround times are often long, and the benefits of diagnosis are dominated by the potential to reduce onward transmission.

\section{REFERENCES}

1. Dan-Nwafor C, Ochu CL, Elimian K, et al. Nigeria's public health response to the COVID-19 pandemic: January to May 2020. J Glob Health. 2020;10:020399.

2. Aziz AB, Raqib R, Khan WA, et al. Integrated control of COVID-19 in resource-poor countries. Int J Infect Dis. 2020;101:98-101.

3. Giri AK, Rana DR. Charting the challenges behind the testing of COVID-19 in developing countries: Nepal as a case study. Biosaf Health. 2020;2:53-56.

4. Woloshin S, Patel N, Kesselheim AS. False negative tests for SARS-CoV-2 infection-challenges and implications. N Engl J Med. 2020;383:e38.

5. West CP, Montori VM, Sampathkumar P. COVID-19 Testing: the threat of false-negative results. Mayo Clin Proc. 2020;95:1127-1129.

6. Larremore DB, Wilder B, Lester E, et al. Test sensitivity is secondary to frequency and turnaround time for COVID-19 screening. Sci Adv. 2020;7:eabd5393.

7. World Health Organization. COVID-19 Target Product Profiles for Priority Diagnostics to Support Response to the COVID-19 Pandemic v. 1.0. 2020. Available at: https://www.who.int/publications $/ \mathrm{m} / \mathrm{item} / \mathrm{covid}-$ 19-target-product-profiles-for-priority-diagnostics-to-support-responseto-the-covid-19-pandemic-v.0.1. Accessed October 25, 2020.

8. US Centers for Diseases Control and Prevention. Interim Guidance for Antigen Testing for SARS-CoV-2. COVID-19 (Coronavirus Disease). 2020. Available at: https://www.cdc.gov/coronavirus/2019-ncov/lab/ resources/antigen-tests-guidelines.html. Accessed December 15, 2020.

9. European Centre for Disease Prevention and Control. Options for the use of rapid antigen tests for COVID-19 in the EU/EEA and the UK. 2020. Available at: https://www.ecdc.europa.eu/sites/default/files/documents/Options-use-of-rapid-antigen-tests-for-COVID-19.pdf. Accessed December 15, 2020.

10. World Health Organization. Antigen-detection in the Diagnosis of SARSCoV-2 Infection Using Rapid Immunoassays: Interim Guidance. 2020. Available at: https://www.who.int/publications/i/item/antigen-detectionin-the-diagnosis-of-sars-cov-2infection-using-rapid-immunoassays. Accessed December 15, 2020.
11. World Health Organization. Global Partnership to Make Available 120 Million Affordable, Quality COVID-19 Rapid Tests for Low-and Middle-Income Countries. 2020. Available at: https://www.who.int/news/ item/28-09-2020-global-partnership-to-make-available-120-million-affordable-quality-covid-19-rapid-tests-for-low--and-middle-income-countries. Accessed October 25, 2020.

12. Vickers AJ, Elkin EB. Decision curve analysis: a novel method for evaluating prediction models. Med Decis Making. 2006;26:565-574.

13. Vickers AJ, van Calster B, Steyerberg EW. A simple, step-by-step guide to interpreting decision curve analysis. Diagn Progn Res. 2019;3:18.

14. Van Calster B, Wynants L, Verbeek JFM, et al. Reporting and interpreting decision curve analysis: a guide for investigators. Eur Urol. 2018;74:796-804.

15. Ritchie H, Ortiz-Ospina E, Beltekian D, et al. The Share of COVID-19 Tests That Are Positive. Our World in Data. Available at: https://ourworldindata.org/grapher/positive-rate-daily-smoothed. Accessed November 10, 2020.

16. Weiner R, Wan W, Hauslohner A. Long delays in getting test results hobble coronavirus response. Washington Post. Available at: https:// www.washingtonpost.com/health/long-delays-in-getting-test-resultshobble-coronavirus-response/2020/07/12/d32f7fa 8-c1 fe-11 ea-b4f6cb39cd8940fb_story.html. Accessed November 10, 2020.

17. Savage M. Coronavirus test results must come in 24 hours, says Sage scientist. The Guardian. 2020. Available at: http://www.theguardian. com/world/2020/oct/18/coronavirus-test-results-must-come-in-24-hourssays-sage-scientist. Accessed November 10, 2020.

18. Sun Y, Koh V, Marimuthu K, et al; National Centre for Infectious Diseases COVID-19 Outbreak Research Team. Epidemiological and clinical predictors of COVID-19. Clin Infect Dis. 2020;71:786-792.

19. He X, Lau EHY, Wu P, et al. Temporal dynamics in viral shedding and transmissibility of COVID-19. Nat Med. 2020;26:672-675.

20. Tindale LC, Stockdale JE, Coombe M, et al. Evidence for transmission of COVID-19 prior to symptom onset. Elife. 2020;9:e57149.

21. Du Z, Xu X, Wu Y, Wang L, Cowling BJ, Meyers LA. Serial interval of COVID-19 among publicly reported confirmed cases. Emerg Infect Dis. 2020;26:1341-1343.

22. Iglói Z, Velzing J, van Beek J, et al. Clinical evaluation of the Roche/SD Biosensor rapid antigen test with symptomatic, non-hospitalized patients in a municipal health service drive-through testing site. [Published online ahead of print January 1, 2020]. medRxiv. doi:10.1101/2020.11.18.20234104.

23. Tenforde MW, Kim SS, Lindsell CJ, et al. Symptom Duration and Risk Factors for Delayed Return to Usual Health Among Outpatients with COVID-19 in a Multistate Health Care Systems Network-United States, March-June 2020. MMWR Morb Mortal Wkly Rep. 2020;69:993-998.

24. Faes C, Abrams S, Van Beckhoven D, Meyfroidt G, Vlieghe E, Hens N; Belgian Collaborative Group on COVID-19 Hospital Surveillance. Time between symptom onset, hospitalisation and recovery or death: statistical analysis of belgian COVID-19 patients. Int J Environ Res Public Health. 2020;17:E7560.

25. Steens A, de Blasio BF, Veneti L, et al. Poor self-reported adherence to COVID-19-related quarantine/isolation requests, Norway, April to July 2020. Eurosurveillance. 2020;25:2001607.

26. Smith LE, Amlôt R, Lambert H, et al. Factors associated with adherence to self-isolation and lockdown measures in the UK; a cross-sectional survey. [Published online ahead of print June 2, 2020]. medRxiv. doi:10.1101/2020.06.01.20119040.

27. Kissler SM, Fauver JR, Mack C, et al. Viral dynamics of SARS-CoV-2 infection and the predictive value of repeat testing. Epidemiology 2020.

28. To KK, Tsang OT, Leung WS, et al. Temporal profiles of viral load in posterior oropharyngeal saliva samples and serum antibody responses during infection by SARS-CoV-2: an observational cohort study. Lancet Infect Dis. 2020;20:565-574.

29. Sia SF, Yan LM, Chin AWH, et al. Pathogenesis and transmission of SARS-CoV-2 in golden hamsters. Nature. 2020;583:834-838.

30. Jefferson T, Spencer E, Brassey J, Heneghan C. Viral cultures for COVID-19 infectivity assessment. Systematic review. [Published online ahead of print September 29, 2020]. medRxiv. doi:10.1101/2020.08.04.20167932.

31. Singanayagam A, Patel M, Charlett A, et al. Duration of infectiousness and correlation with RT-PCR cycle threshold values in cases of COVID-19, England, January to May 2020. Euro Surveill. 2020;25:2001483.

32. Smith RL, Gibson LL, Martinez PP, et al. Longitudinal assessment of diagnostic test performance over the course of acute 
SARS-CoV-2 infection. medRxiv. Published online March 22, 2021. doi: 10.1101/2021.03.19.21253964

33. Berger A, Ngo Nsoga MT, Perez-Rodriguez FJ, et al. Diagnostic accuracy of two commercial SARS-CoV-2 Antigen-detecting rapid tests at the point of care in community-based testing centers. [Published online ahead of print November 23, 2020]. medRxiv. doi:10.1101/2020.11.20.20235341.

34. Kucirka LM, Lauer SA, Laeyendecker O, Boon D, Lessler J. Variation in falsenegative rate of reverse transcriptase polymerase chain reaction-based SARSCoV-2 tests by time since exposure. Ann Intern Med. 2020;173:262-267.

35. Fitzgerald M, Saville BR, Lewis RJ. Decision curve analysis. JAMA. 2015;313:409-410.

36. Vickers AJ, Van Calster B, Steyerberg EW. Net benefit approaches to the evaluation of prediction models, molecular markers, and diagnostic tests. BMJ. 2016;352:i6.

37. Chin ET, Huynh BQ, Chapman LAC, Murrill M, Basu S, Lo NC Frequency of routine testing for coronavirus disease 2019 (COVID-19) in high-risk healthcare environments to reduce outbreaks [published online ahead of print October 26, 2020]. Clin Infect Dis. doi: 10.1093/ $\mathrm{cid} / \mathrm{ciaa} 1383$

38. Paltiel AD, Zheng A, Walensky RP. Assessment of SARS-CoV-2 screening strategies to permit the safe reopening of college campuses in the United States. JAMA Netw Open. 2020;3:e2016818.

39. Losina E, Leifer V, Millham L, et al. College campuses and COVID19 mitigation: clinical and economic value. Ann Intern Med. 2021;174:472-483.

40. Lai SHS, Tang CQY, Kurup A, Thevendran G. The experience of contact tracing in Singapore in the control of COVID-19: highlighting the use of digital technology. Int Orthop. 2020;45:65-69.

41. Endo A, Leclerc QJ, Knight GM, et al; Centre for the Mathematical Modelling of Infectious Diseases COVID-19 Working Group. Implication of backward contact tracing in the presence of overdispersed transmission in COVID-19 outbreaks. Wellcome Open Res. 2020;5:239. 